\title{
Phospholipid homeostasis plays an important role in fungal development, fungicide resistance and virulence in Fusarium graminearum
}

\author{
Jing Wang ${ }^{1}$, Haixia Wang ${ }^{1}$, Chenying Zhang ${ }^{1}$, Tianjing $\mathrm{Wu}^{1}$, Zhonghua $\mathrm{Ma}^{1,2,3}$ and Yun Chen ${ }^{1,2^{*}}$ (i)
}

\begin{abstract}
Phospholipids are major structural components of all cell membranes and participate in energy storage, signal transduction and environmental adaptability in eukaryotes. To date, the enzymes involved in phospholipid biosynthesis have been well characterized in budding yeast. However, their functions in filamentous fungi are largely unclear, especially their contribution to the interaction between phytopathogenic filamentous fungi and plants. In this study, we identified 10 phospholipid biosynthesis-related genes and genetically analyzed their functions in the Fusarium head blight pathogen Fusarium graminearum. The results of this study indicate that phosphatidylethanolamine (PE) and phosphatidylcholine (PC) are critical for fungal vegetative growth. The biosynthesis of PE and PC is largely dependent on FgPsd2, FgCho2 and FgOpi3 in the de novo pathway of phospholipid biosynthesis in F. graminearum. Phospholipid biosynthetic gene mutants showed abnormal conidiation, increased sensitivity to fungicides and the oxidative stress agent $\mathrm{H}_{2} \mathrm{O}_{2}$, and defective endocytosis, especially the $\Delta$ Fgpsd2, $\Delta F g c h o 2$ and $\Delta$ Fgopi3 mutants. Importantly, this study shows for the first time that the de novo pathway of phospholipid biosynthesis is required for mycotoxin production and full virulence in plant pathogenic fungi.
\end{abstract}

Keywords: Fusarium graminearum, Fungicide resistance, Pathogenicity, The de novo pathway of phospholipid biosynthesis

\section{Background}

A phospholipid bilayer with embedded, integral and peripheral proteins constitutes the basic skeleton of the cell membrane, which acts as a selectively permeable barrier to isolate the cell from the external environment and ensure the independence of biochemical reactions in living organisms. Phospholipids can be further divided into phosphatidylcholine (PC), phosphatidylethanolamine (PE), phosphatidylinositol (PI) and phosphatidylserine (PS) based on their head groups (Nagle and Tristram-Nagle 2000). In eukaryotes and some prokaryotes, PE and PC are

\footnotetext{
* Correspondence: chenyun0927@zju.edu.cn

${ }^{1}$ Institute of Biotechnology, Zhejiang University, 866 Yuhangtang Road, Hangzhou 310058, China

${ }^{2}$ Key Laboratory of Molecular Biology of Crop Pathogens and Insects, Zhejiang University, 866 Yuhangtang Road, Hangzhou 310058, China Full list of author information is available at the end of the article
}

the most abundant phospholipids, accounting for more than $50 \%$ of the total phospholipid population (Vance and Steenbergen 2005).

Routes of PE and PC biosynthesis have been thoroughly investigated in the baker's yeast Saccharomyces cerevisiae. In yeast, $\mathrm{PE}$ and $\mathrm{PC}$ are synthesized via both endogenous (the de novo pathway) and exogenous pathways (the Kennedy pathway) (Daum et al. 1998; Gibellini and Smith 2010; Cassilly and Reynolds 2018). PS is synthesized from cytidyldiphosphate-diacylglycerol (CDP-DAG) and serine by the enzyme Cho1 with the associated release of cytidylmonophosphate (CMP) (Letts et al. 1983). Subsequently, PS is decarboxylated by phosphatidylserine decarboxylase 1 (Psd1p) localized in the inner mitochondrial membrane, and Golgi-localized phosphatidylserine decarboxylase 2 (Psd2p) to generate PE (Zinser et al. 1991; Trotter and Voelker 1995). Then, PE is methylated by two types of

(c) The Author(s). 2019 Open Access This article is distributed under the terms of the Creative Commons Attribution 4.0 International License (http://creativecommons.org/licenses/by/4.0/), which permits unrestricted use, distribution, and reproduction in any medium, provided you give appropriate credit to the original author(s) and the source, provide a link to the Creative Commons license, and indicate if changes were made. The Creative Commons Public Domain Dedication waiver (http://creativecommons.org/publicdomain/zero/1.0/) applies to the data made available in this article, unless otherwise stated. 
phospholipid methyltransferases, Cho2 and Opi3, using S-adenosyl-L-methionine as the methyl donor to yield PC (Daum et al. 1998; Gibellini and Smith 2010).

However, the Kennedy pathway incorporates exogenous ethanolamine (Etn) or choline (Cho) directly into PE or PC, respectively. Exogenous Etn and Cho are phosphorylated by the kinases Eki1 and Cki to generate phosphoethanolamine (EtnP) and phosphocholine (ChoP). EtnP and ChoP are subsequently catalyzed by the cytidylyltransferases Ect1 and Pct1, respectively, to generate cytidine diphosphate ethanolamine (CDP-Etn) and cytidine diphosphocholine (CDP-Cho). Finally, CDP-Etn and CDP-Cho react with DAG to form PE and PC, respectively (Birner et al. 2001). Mammals share conserved phospholipid biosynthesis pathways with yeast, but a major difference is found in PS biosynthesis (Kent 1995). In mammals, two phosphatidylserine synthases, Pss1 and Pss2, exchange serine for Etn in PE or for Cho in PC to generate PS, unlike the Cho1-dependent process of PS synthesis in yeast (Sturbois-Balcerzak et al. 2001; Bergo et al. 2002). A genomic survey indicates that Cho1, which has been suggested as a potential antifungal drug target, is conserved in fungi but absent in mammals (Braun et al. 2005).

In addition to their role in the formation of membrane bilayers, phospholipid biosynthetic genes also directly or indirectly participate in cytokinesis, cell wall integrity, secretion and several other biological processes (Wolf et al. 2015). For instance, PS synthase is required for the proper regulation of cellular morphogenesis and cytokinesis in Schizosaccharomyces pombe. Deletion of the PS synthase gene caused cell necrosis and severe morphological and cytokinesis abnormalities, including bent, bulbous, branched, and/or ovoid cells (Matsuo et al. 2007). The Candida albicans $\Delta / \Delta$ chol mutant exhibits defects in cell wall integrity and mitochondrial function (Chen et al. 2010). The $\Delta / \Delta c h o 1$ and $\Delta / \Delta p s d 1 \Delta / \Delta p s d 2$ strains showed defects in extracellular vesicle morphology and cargo, and immunostimulatory properties (Wolf et al. 2015). Moreover, accumulating evidence suggests that phospholipids are also important for the interaction of symbiotic and pathogenic microorganisms with host cells. The PC level in Bradyrhizobium japonicum membranes is critical for efficient symbiosis with the soybean host plant (Minder et al. 2001). Disruption of the PS synthase Pssa impaired several virulence traits in Brucella abortus, such as intracellular survival in macrophages and HeLa cells, maturation of the replicative Brucella-containing vacuole, and mouse colonization (Bukata et al. 2008). Agrobacterium tumefaciens mutants unable to synthesize PC showed a defective type IV secretion system and impaired tumor formation on Kalanchoë plants (Wessel et al. 2006). Both PS synthesis and de novo PE synthesis are required for C. albicans virulence (Chen et al. 2010). However, little is known about the functions of phospholipids in filamentous fungi, especially their contribution to the interaction between pathogenic filamentous fungi and host cells.

The filamentous fungus Fusarium graminearum is the predominant pathogenic agent of the devastating Fusarium head blight (FHB) disease of wheat (Xu and Nicholson 2009). Epidemics and the incidence of FHB directly cause severe yield losses in FHB-prone regions worldwide. For example, an FHB epidemic with a high incidence rate has resulted in the infection of approximately $20 \%$ of the planting area of wheat since 2010 in China (Chen et al. 2017). More critically, F. graminearum produces harmful mycotoxins, such as deoxynivalenol (DON), nivalenol and zearalenone, in infested grains (Tang et al. 2018a). Currently, the application of chemical fungicides remains the main approach to control FHB due to the unavailability of FHB-resistant wheat cultivars (Blandino et al. 2012). Unfortunately, fungicide-resistant $F$. graminearum strains have been detected in the field after intensive applications of fungicides (Zhang et al. 2013). Moreover, the application of several fungicides, including carbendazim and azoles, at sublethal concentrations triggers DON biosynthesis (Milus and Parsons 1994; Simpson et al. 2001; Magan et al. 2002; Zhang et al. 2009; Audenaert et al. 2010; Tang et al. 2018a). Therefore, the identification of potential drug targets for the development of new fungicides is imperative.

In this study, we identified phospholipid biosynthetic genes and genetically analyzed their functions in F. graminearum. The results of this study indicate that $\mathrm{PE}$ and PC are critical for mycelial growth and that the biosynthesis of PE and PC is largely dependent on the de novo pathway in F. graminearum. Importantly, this study shows for the first time that the de novo pathway of phospholipid synthesis participates in the mycotoxin production and full virulence of this important phytopathogenic fungus.

\section{Results \\ Identification of genes participating in PE and PC biosynthesis}

The sequences of all enzymes directly involved in the PE and PC biosynthetic pathway in budding yeast were used as queries for BlastP searches to identify homologous genes in the genome of $F$. graminearum. A total of 10 proteins were identified, and their functions in F. graminearum were predicted (Table 1). Next, these proteins were assigned to the locations of their yeast counterparts (Fig. 1a) based on the biosynthetic pathway of PE and PC in yeast. Although the identified proteins involved in PE and PC biosynthesis in F. graminearum shared relatively low identity (31\%$54 \%$ ) with their homologs (Table 1), structural analysis revealed that these proteins contained the same functional 
Table 1 Predicted proteins involved in the PE and PC biosynthesis pathway in F. graminearum

\begin{tabular}{|c|c|c|c|c|}
\hline Protein in yeast & Function & Homologues in F.graminearum & Identities (\%) & E-value \\
\hline Cho1 & CDP-diacylglycerol serine O-phosphatidyltransferase & FgCho1 (FGSG_06370) & 54 & $3.6 \mathrm{e}-61$ \\
\hline Psd1 & Phosphatidylserine decarboxylase in the mitochondrion & FgPsd1(FGSG_01865) & 46 & $4.1 \mathrm{e}-92$ \\
\hline Psd2 & Phosphatidylserine decarboxylase 2 & FgPsd2 (FGSG_10007) & 50 & $1.6 \mathrm{e}-102$ \\
\hline Cho2 & Phosphatidyl-ethanolamine methyltransferase & FgCho2 (FGSG_05066) & 32 & $5.3 e-104$ \\
\hline Opi3 & Methylene-fatty-acyl-phospholipid synthase & FgOpi3 (FGSG_08613) & 51 & $1.0 \mathrm{e}-55$ \\
\hline Eki1/Cki1 & Ethanolamine kinase/ choline kinase & FgCki1 (FGSG_17231) & 31 & $3.4 \mathrm{e}-38$ \\
\hline Ect1 & Ethanolamine-phosphate cytidylyltransferase & FgEct1 (FGSG_04571) & 32 & $2.8 \mathrm{e}-43$ \\
\hline Ept1 & Ethanolamine phosphotransferase & FgEpt1 (FGSG_08706) & 37 & $6.2 \mathrm{e}-67$ \\
\hline Pct1 & Choline-phosphate cytidylyltransferase & FgPct1(FGSG_10005) & 54 & $4.5 e-81$ \\
\hline Cpt1 & Cholinephosphotransferase & FgCpt1 (FGSG_09402) & 35 & $1.3 e-48$ \\
\hline
\end{tabular}

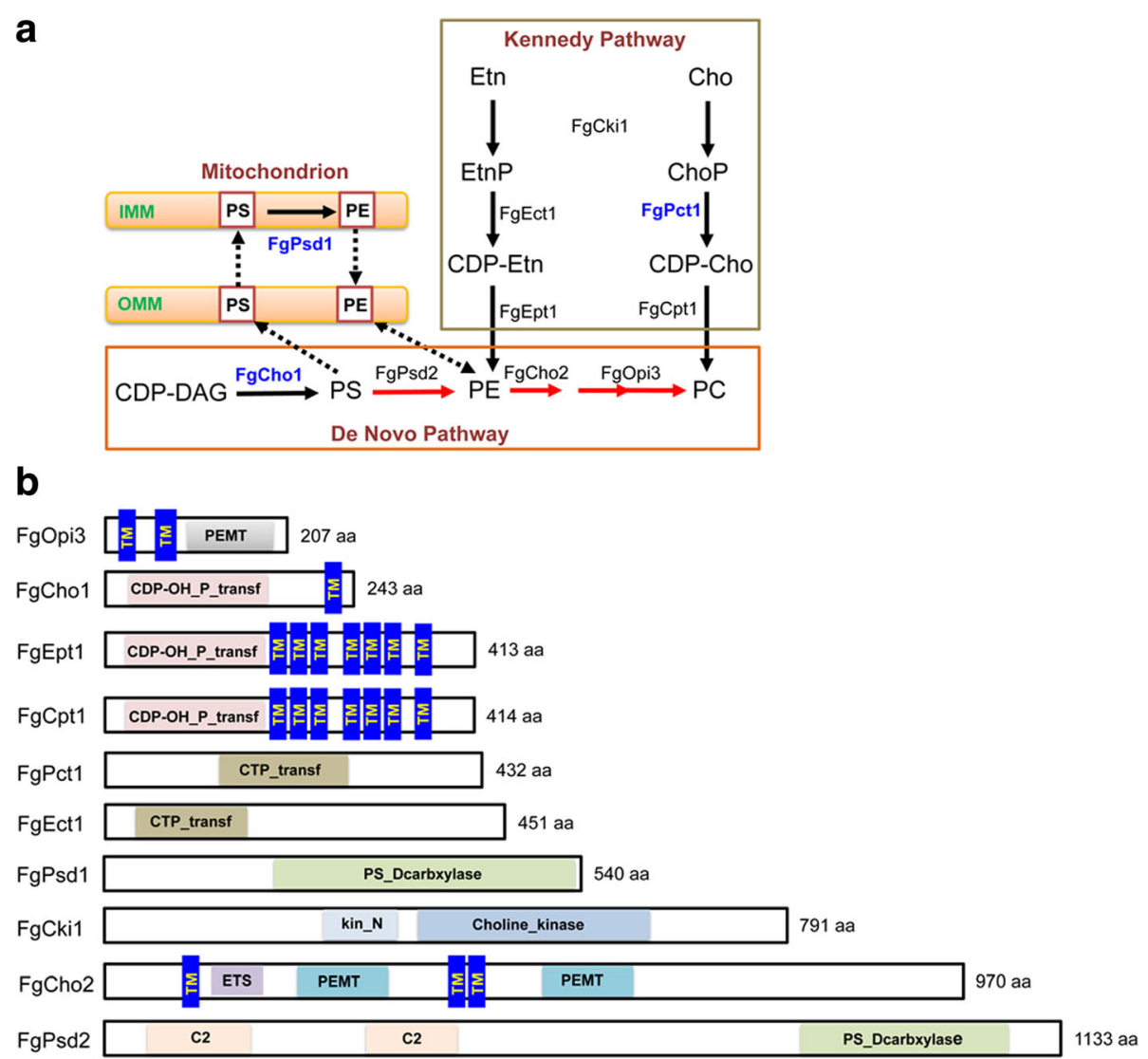

Fig. 1 Identification of phospholipid biosynthetic genes in Fusarium graminearum. a Schematic outline of phospholipid biosynthesis pathways in F. graminearum. Two alternative pathways of phospholipid biosynthesis, the de novo pathway and the Kennedy pathway, exist in this fungus. The essential enzymes are marked in blue. The major routes for PE and PC biosynthesis are indicated by the red arrows. FgPsd1 is predicted to be localized on the inner mitochondrial membrane (IMM). b Predicted conserved domains in the phospholipid biosynthetic enzymes of $F$. graminearum. OMM, outer mitochondrial membrane; CDP-DAG, cytidyldiphosphate diacylglycerol; PS, phosphatidylserine; PE, phosphatidylethanolamine; $P C$, phosphatidylcholine; Etn, ethanolamine; EtnP, ethanolamine phosphate; CDP-Etn, cytidyldiphosphate-ethanolamine; Cho, choline; ChoP, choline phosphate; CDP-Cho, cytidinediphosphocholine; TM, transmembrane; PEMT, phospholipid methyltransferase; CDP-OH_P_transf, CDP-alcohol phosphatidyltransferase; Ctp_transf, cytidylyltransferase; PS_Dcarbxylase, phosphatidylserine decarboxylase-related; kin_N, choline kinase N terminus; ETS, erythroblast transformation specific domain; $C 2$, protein kinase $C$ conserved region 2 
domains as their yeast counterparts (Fig. 1b). The kinase Eki1 and its paralog Ckil phosphorylate ethanolamine and choline, respectively, in the yeast Kennedy pathway. However, F. graminearum FGSG_17231 is homologous to both yeast Eki1 and Cki1, which indicates that the protein encoded by the FGSG_17231 locus may have both ethanolamine kinase and choline kinase activity; this protein is hereafter designated FgCkil. Taken together, the data from the bioinformatic analysis suggest that proteins participating in PE and PC biosynthesis are relatively conserved in yeast and filamentous fungi, at least in $F$. graminearum.

Biosynthesis of PE and PC is largely dependent on the de novo pathway

To characterize the function of the above 10 identified PE and PC biosynthetic genes, we generated deletion mutants of each gene using the homologous recombination approach. After the screening step, hygromycin-resistant transformants were identified as successful deletion mutants by polymerase chain reaction (PCR) amplification with the primer pair P5 + P6 (Additional file 1: Table S1) and further analyzed via a Southern blot assay (Additional file 2: Figure S1). Among these mutants, 7 genes- $F g P S D 2, F g C H O 2$, FgOPI3, FgECT1, FgEPT1, FgCKI1 and FgCPT1-were successfully deleted individually. For $\mathrm{FgCHO1}, \mathrm{FgPSD} 1$ and $F g P C T 1$, we failed to identify true gene knockout mutants after screening over 100 ectopic transformants from at least three independent transformation experiments, indicating that the deletion of these genes may be lethal because of the high homologous recombination efficiency in F. graminearum (Yun et al. 2015).

All resulting gene deletion mutants were characterized for defects in mycelial growth on various media. The phenotypes of at least three knockout mutants of each gene were examined. As shown in Fig. 2 and Additional file 3: Figure S2, the hyphal growth rate of each mutant was not significantly different from that of the wild-type $\mathrm{PH}-1$ strain on complete medium (CM), except for $\Delta$ Fgect1 and $\Delta$ Fgcki1, which exhibited a $15 \%$ reduction in growth. Mutants in which the single circuitry of the Kennedy pathway was disrupted-Etn-EtnP-CDPEtn-PE (AFgect1 and $\Delta$ Fgept 1$)$ or Cho-ChoP-CDPCho-PC

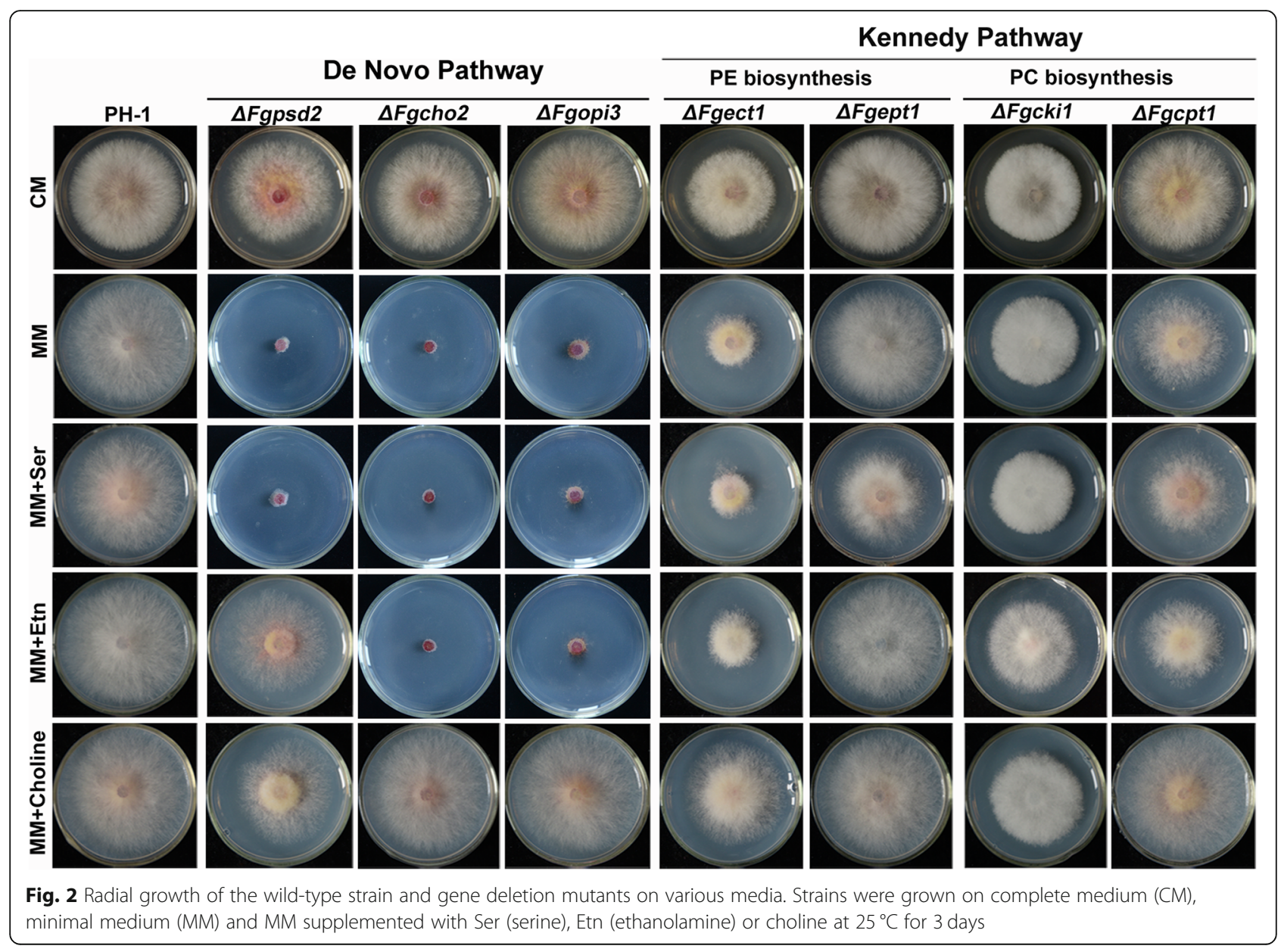


( $\triangle F g c k i 1$ and $\Delta F g c p t 1)$-were able to grow on minimal medium (MM). However, all three deletions of genes in the de novo pathway of phospholipid biosynthesis (the $\Delta F g p s d 2, \Delta F g c h o 2$ and $\Delta F g o p i 3$ mutants) almost abolished vegetative growth on MM.

To test whether the growth phenotypes of the mutants were caused by defects in PE or PC, MM was supplemented individually with the initial precursors in different subpathways-serine, ethanolamine and choline-to rescue the defects. Clearly, supplementation with serine was not able to rescue the growth defects of the mutants. This result suggested that FgPsd1 played a minor role in PE biosynthesis and could not serve as an alternative PE source to support mycelial growth in the absence of FgPSD2. In addition, the growth defects of $\Delta F g p s d 2$ but not $\triangle F g c h o 2$ and $\Delta$ Fgopi3 were partially compensated by the addition of exogenous ethanolamine, which was presumed to increase the amount of cellular PE through the Etn-EtnP-CDPEtn-PE process in the Kennedy pathway (Fig. 1a). Notably, the increased amount of PC generated by supplementation with choline rescued the growth defects in all mutants to different extents. Taken together, these findings indicate that the biosynthesis of PE and PC largely depends on the de novo pathway. Moreover, the de novo pathway is critical for hyphal growth in $F$. graminearum.

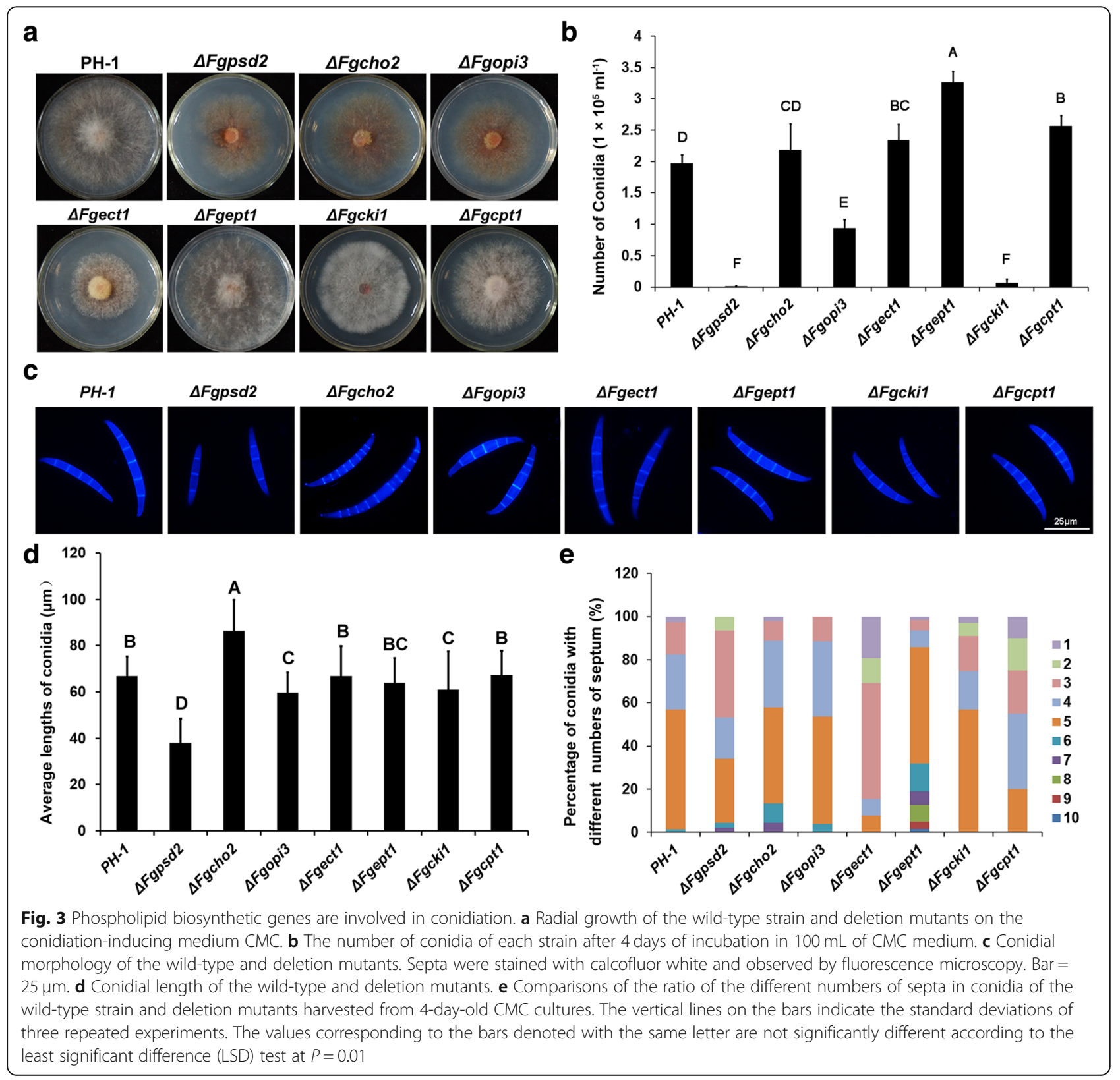


PE and PC biosynthetic genes are involved in conidiation To determine the role of PE and PC biosynthetic genes in asexual reproduction, the number of conidia generated by the individually tested strains in carboxymethyl cellulose (CMC) medium was calculated. All strains were able to grow on the CMC agar plates (Fig. 3a). After 4 days of incubation, the wild-type strain produced approximately $2 \times 10^{5}$ conidia/mL, while the $\Delta F g p s d 2$, $\triangle F g o p i 3$ and $\Delta F g c k i 1$ strains produced significantly fewer conidia than the wild-type strain. In contrast, the $\Delta$ Fgept 1 strain produced $3.3 \times 10^{5}$ conidia/mL, significantly more than the wild-type strain (Fig. 3b). To further examine the conidial morphology, conidia were stained with calcofluor white and observed under a fluorescence microscope. As shown in Fig. 3c-e, the average size of conidia produced by the $\Delta$ Fgps 22 strain was $38 \pm 9.4 \mu \mathrm{m}$, which was smaller than that of the wild-type strain $(67 \pm 8.7 \mu \mathrm{m})$. Moreover, conidia of the $\Delta$ Fgpsd2 strain exhibited fewer septa than conidia of the wild-type strain. Most of the conidia (approximately 54\%) produced by the mutant strains had three or fewer septa, whereas the majority of conidia produced by the wild-type strain had five septa (Fig. 3b-d). Interestingly, the $\Delta F g c h o 2$ deletion mutant produced larger conidia $(86 \pm 7.8 \mu \mathrm{m})$ with more septa than the wild-type strain. Over $30 \%$ of these conidia contained more than five septa, or even ten septa. In the complemented strains $\triangle F g p s d 2-C, \triangle F g c h o 2-C$ and $\triangle F g o-$ pi3- $C$, the conidia production and morphological defects of the corresponding mutants were rescued (Additional file 4: Figure S3a-c).
Mutants of the de novo pathway show increased sensitivity to multiple antifungal agents

In previous studies, it has been reported that membrane lipids are involved in drug susceptibility, cold stress and other abiotic stresses (Welti et al. 2002; Gulshan et al. 2010; Khandelwal et al. 2018). Therefore, we were interested in determining the susceptibility of the $F$. graminearum mutants to various stresses, including fungicide treatment, osmotic stress and cell wall stress. As shown in Fig. 4a and b, the $\Delta F g p s d 2, \Delta F g c h o 2$ and $\Delta F g o p i 3$ mutants exhibited significantly increased sensitivity to rapamycin, carbendazim, phenamacril and tebuconazole. Among the mutants, the $\Delta F g p s d 2$ mutant exhibited the highest sensitivity to the four tested fungicides. However, the $\Delta F g p s d 2$ mutant grew similar to the wild-type strain in medium supplemented with osmotic or cell wall stress agents (data not shown). Reintroduction of the wild-type allele restored the sensitivity of all three mutants towards the tested fungicides to that of the PH-1 strain (Additional file 4: Figure S3d, e).

\section{The de novo pathway of phospholipid biosynthesis is required for full virulence}

The virulence of each mutant was evaluated by point inoculation of a conidial suspension on flowering wheat heads. On wheat heads inoculated with the wild-type strain PH-1, scab symptoms first developed on the inoculated spikelets and rapidly spread to the neighboring spikelets, causing serious and typical Fusarium head blight symptoms after two weeks of inoculation. However, under the same conditions, the mutants of the de

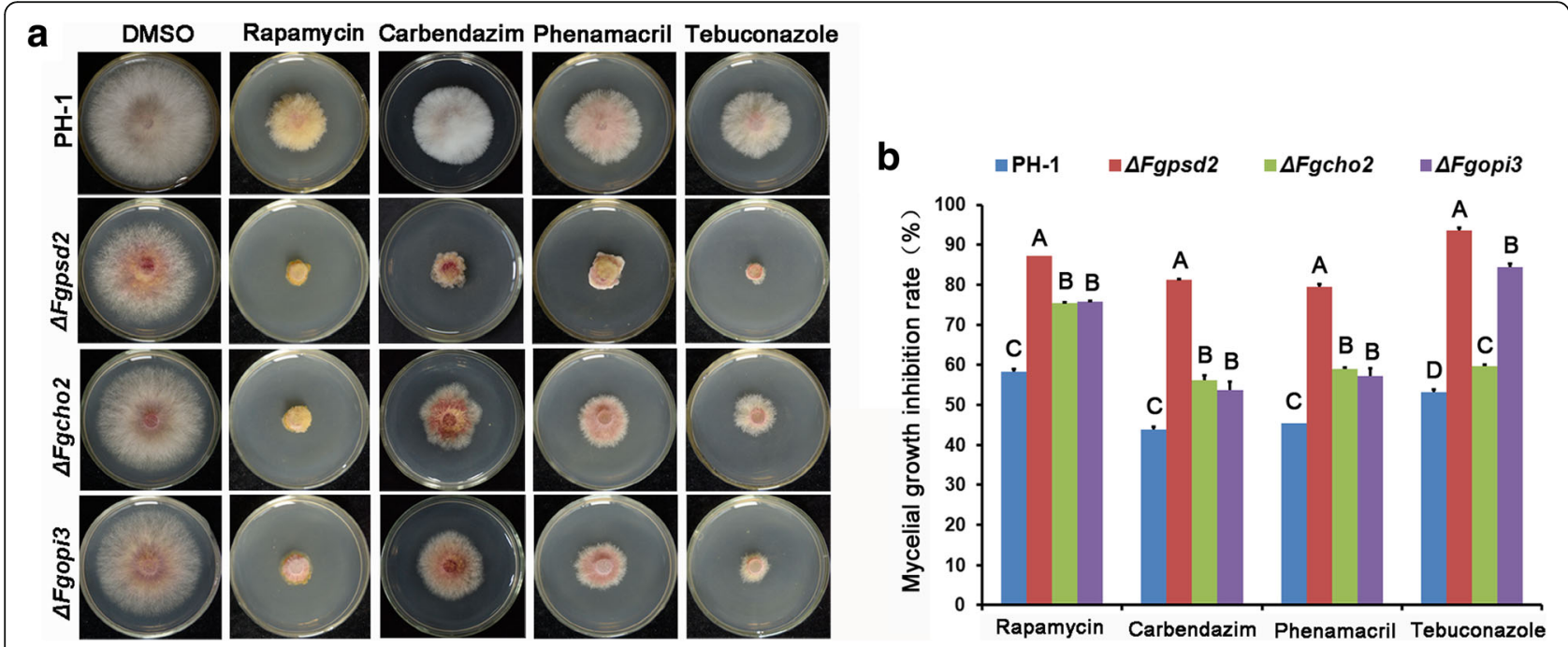

Fig. 4 Disruption of phospholipid biosynthetic genes leads to increased sensitivity to multiple antifungal agents. a Inhibition of mycelial growth of the wild-type and mutant strains by each antifungal agent. Strains were grown on CM amended without or with tebuconazole, carbendazim, phenamacril or rapamycin at the concentration indicated in the methods section. Images were obtained when the mycelia of the wild-type strain reached the edge of the plate. $\mathbf{b}$ Percentage of mycelial growth inhibition by each antifungal agent in each strain. The vertical lines on the bars in each column denote the standard deviations of three repeated experiments. The values corresponding to the bars denoted with the same letter are not significantly different according to the (LSD) test at $P=0.01$ 


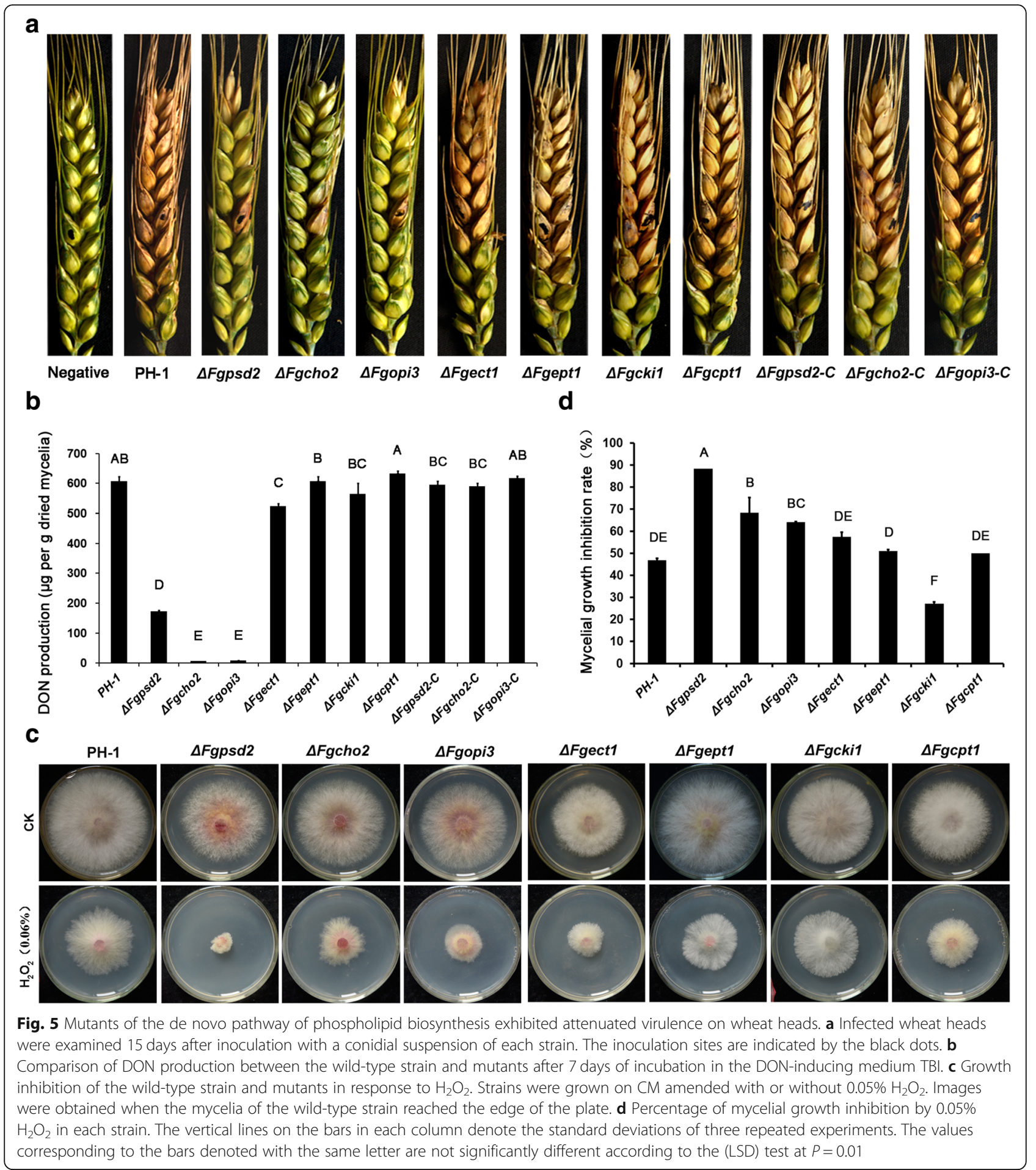

novo pathway ( $\triangle F g p s d 2, \Delta F g c h o 2$ and $\Delta F g o p i 3$ ) failed to spread from the inoculated floret to the rachis and caused scab symptoms only in the inoculated spikelets (Fig. 5a).

DON is an important virulence factor and plays critical roles in the spread of the fungus within host tissues (Proctor et al. 1995; Desjardins et al. 1996). Since the deletion mutants of the de novo pathway attenuated disease spread in planta, DON production was examined. As expected, these three mutants produced significantly less DON than the wild-type strain after 7 days of incubation in trichothecene biosynthesis induction (TBI) medium (Fig. 5b). In addition, these three mutants were 
more sensitive than the wild-type strain to $\mathrm{H}_{2} \mathrm{O}_{2}$, which is a common oxidative agent generated by host plants during fungal infection. Complemented strains of these three mutants and other mutants in the Kennedy pathway produced similar amounts of DON and caused the same disease severity on wheat heads (Fig. 5). Collectively, these findings indicate that the de novo pathway of phospholipid biosynthesis plays an important role in the full virulence of F. graminearum.

\section{Disruption of phospholipid synthesis impairs fungal endocytosis}

The phospholipid composition of cellular membranes has been suggested to be required for the intracellular trafficking of some transporters (Guo et al. 2010; Lee et al. 2012). The endocytosis of mutants was therefore examined using a FM4-64 staining assay. The plasma membrane and septa were quickly stained by FM4-64 within $1 \mathrm{~min}$ in both the wild-type strain and the mutants. FM4-64 dye was taken up, and a fluorescence signal clearly appeared on the plasma-facing membrane of intracellular organelles, such as vacuoles and endosomes, after $5 \mathrm{~min}$ of staining in the mycelia of the wild-type strain. However, the uptake of the fluorescent dye was appreciably impaired in all mutants tested, even after $10 \mathrm{~min}$ of incubation (Fig. 6). These results suggest that the biosynthesis of PS, $\mathrm{PE}$ and $\mathrm{PC}$ is important for normal endocytosis.

\section{Discussion}

Lipids are important biomolecules and include phospholipids, fatty acids, sterols, and other molecules. The functions of lipids include storing energy, signaling, and acting as structural components of cell membranes (Subramaniam et al. 2011). Cells use $\sim 5 \%$ of their genes to synthesize lipids for cell viability, suggesting the complexity and importance of lipids (Sud et al. 2007; Van Meer et al. 2008). Because of this complexity and the differences in lipid biosynthetic and metabolic pathways between fungal and mammalian cells, the key enzymes in these pathways have been suggested to be targets of fungicides (Pan et al. 2018). For instance, azoles, polyene macrolides and other inhibitors have been developed to interfere with ergosterol biosynthesis to suppress fungal infection (Ghannoum and Rice 1999). Phospholipids are critical structural components of cell membranes and are important for fungal growth. In C. albicans, interruption of the de novo pathway of phospholipid biosynthesis by knocking out $\mathrm{CHO} 1$ or double mutation of PSD1/PSD2 caused defects in cell wall integrity, mitochondrial function, filamentous growth and virulence (Chen et al. 2010; Wolf et al. 2015; Cassilly and Reynolds 2018). The $S$. cerevisiae $\Delta p s d 1$ deletion mutant with a blockade of the major PE synthesis pathway was unable to be cultivated on lactate or ethanol without supplementation with Etn, Cho or Ser (Birner et al. 2001). In the filamentous fungi Aspergillus nidulans and Pestalotiopsis microspora, the loss of $\mathrm{ChoC}$ (Opi3 in yeast) resulted in defects in $\mathrm{PC}$ production, vegetative growth and

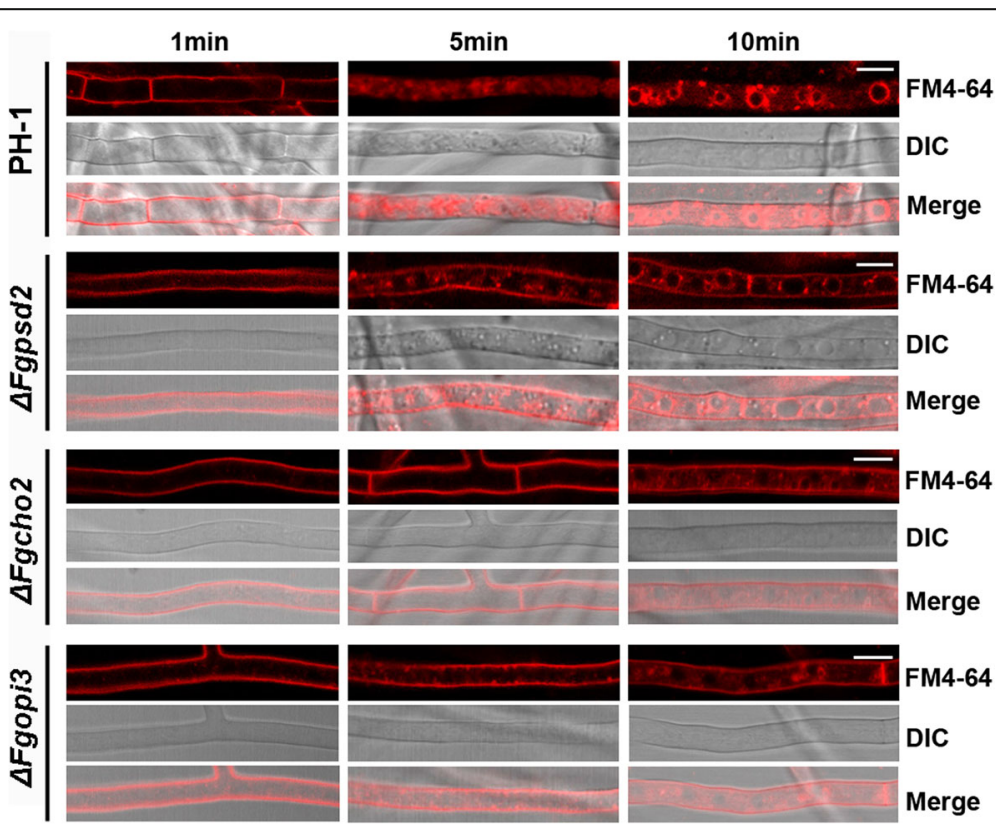

Fig. 6 Deletion mutants of the de novo pathway exhibited impaired fungal endocytosis. Mycelia of the wild-type strain and mutants were stained with FM4-64 and observed via microscopy. Images were acquired at 1, 5 and 10 min after staining. Bar = $20 \mu m$ 
conidiation (Tao et al. 2010; Akhberdi et al. 2018). More importantly, Cho1 and Opi3 (ChoC) do not have homologs in mammalian cells. Therefore, new drugs targeting Cho1 and Opi3 could potentially be developed for antifungal therapy or fungal disease control (Pan et al. 2018). In agreement with this concept, 7-chloro-N-(4-propylphenyl)-4-quinolinamine

(7CPQA) has been shown to reduce Plasmodium yoelii infection in mice, with low toxicity towards mammalian cells, via the inhibition of PSD activity.

In this study, 10 yeast homologous genes involved in PS, $\mathrm{PE}$ and $\mathrm{PC}$ biosynthesis were identified in $F$. graminearum (Fig. 1 and Table 1). Genetic evidence indicates that the de novo pathway is the major route for PS, PE and PC biosynthesis in this fungus. Consistent with the results of previous studies, the de novo pathway was essential for cell growth in minimal medium and participated in mediating asexual development and virulence in $F$. graminearum (Fig. 2, Fig. 3 and Fig. 5). These findings imply that a new antifungal drug targeting a key enzyme in the de novo pathway may be used for FHB disease management in the future. Our results also provide additional evidence to support the hypothesis that the inhibition of Cho1 or Opi3 (ChoC) activity is likely to have a broad-spectrum antifungal effect.

In S. cerevisiae, Psd1 is a major catalytic enzyme of PE formation, which accounts for approximately $80 \%$ of PSD activity in vivo. Loss of Psd2p activity does not affect cell growth (Burgermeister et al. 2004). In contrast, the $\Delta F g p s d 2$ deletion mutant of $F$. graminearum was not able to grow on minimal medium without supplementation with Etn or Cho (Fig. 2), indicating that the PE deficiency in the $\Delta$ Fgpsd2 mutant cannot be rescued by the activity of FgPsd1 and that FgPsd2 is the major enzyme of PC and PE formation in F. graminearum. In addition, we failed to generate the FgPSD1, FgCHO1 and FgPCT1 gene mutants even with high homologous recombination efficiency in $\mathrm{F}$. graminearum, indicating that these genes may be essential for growth and have biological functions in addition to phospholipid synthesis. However, their yeast counterparts are not essential. Collectively, the evidence presented here suggests that partial variation in the metabolic network of phospholipids exists between yeasts and filamentous fungi.

The close link between phospholipid homeostasis and drug resistance has been demonstrated. Overproduction of Psd1 induced the transcription of the gene encoding the ATP-binding cassette transporter Pdr5 and increased cycloheximide resistance in yeast (Gulshan et al. 2008). The $C$. albicans phospholipid mutants $\Delta / \Delta$ chol and $\Delta /$ $\Delta p s d 1 / 2$ showed increased sensitivity to farnesol (Hasim et al. 2018). Recently, Khandelwal et al. (2018) reported that the $\Delta / \Delta p s d 1 \Delta / \Delta p s d 2$ mutant showed increased membrane fluidity and a reduced plasma membrane dipole potential, which subsequently resulted in increased drug accumulation and azole susceptibility in C. albicans. Similarly, dysfunction of phospholipid homeostasis in F. graminearum induced by disrupting biosynthetic genes, especially $F g P S D 2$, also resulted in increased sensitivity to multiple tested fungicides (Fig. 4). Further studies will analyze membrane fluidity and the transcription of multidrug transporters in $\Delta$ Fgpsd 2 to reveal the mechanism of multidrug sensitivity. Therefore, this line of evidence indicates that altering phospholipid homeostasis is likely to be an alternative approach to manage multidrug resistance problems in clinical and agricultural fungal infections and diseases.

Phospholipid biosynthesis is also important for virulence in pathogenic fungi. The concentration of $\mathrm{PC}$ was higher in virulent strains than in an avirulent strain of Paracoccidioides brasiliensis (Vallejo et al. 2012). Defects in phospholipid biosynthesis resulted in attenuated virulence in $C$. albicans and the insect pathogenic fungus Metarhizium robertsii (Chen et al. 2010; Gao et al. 2016; Chen et al. 2018b). Here, we found that the major phospholipid biosynthetic gene mutants, including $\triangle F g p s d 2, \Delta F g c h o 2$ and $\Delta F g o p i 3$, exhibited dramatically attenuated virulence in planta (Fig. 5a). The markedly reduced invasive growth of the mutants was caused by defects in multiple virulence factors. All three mutants exhibited significantly reduced production of DON (Fig. 5b), which has been identified as a critical virulence factor and plays a significant role in the spread of the fungus within wheat spikes (Proctor et al. 1995; Desjardins et al. 1996; Seong et al. 2009). Moreover, disruption of the de novo pathway led to increased sensitivity of $F$. graminearum to oxidative stress. It is well known that the earliest defense event in response to fungal infection, including in the Fusarium-host interaction, is the production of reactive oxygen species (ROS), such as $\mathrm{H}_{2} \mathrm{O}_{2}$, by the plant cell (Lehmann et al. 2015). The results of our previous studies also demonstrated that tolerance to $\mathrm{H}_{2} \mathrm{O}_{2}$ is important for $F$. graminearum pathogenicity (Chen et al., 2018a; Tang et al., 2018b). In addition, the reduction in the growth of the mutants may be responsible for their attenuated virulence. In conclusion, defects in growth, ROS tolerance and DON production contribute to the attenuated virulence of $F$. graminearum mutants of the de novo pathway to host plants.

\section{Conclusions}

The results of this study indicate that PE and PC biosynthesis are largely dependent on the de novo pathway of phospholipid biosynthesis in F. graminearum. Phospholipid biosynthetic genes, especially genes in the de novo pathway of phospholipid biosynthesis, are required for fungal development, abiotic stress adaptation, mycotoxin production and full virulence in this plant pathogenic fungus. 


\section{Methods}

\section{Strains and culture conditions}

The F. graminearum wild-type strain $\mathrm{PH}-1$ was used as the parental strain for transformation experiments. The wild-type strain and the mutants generated in this study were grown on minimal medium (MM) $\left[10 \mathrm{mM} \mathrm{K}_{2} \mathrm{HPO}_{4}\right.$, $10 \mathrm{mM} \mathrm{KH}_{2} \mathrm{PO}_{4}, 4 \mathrm{mM}\left(\mathrm{NH}_{4}\right)_{2} \mathrm{SO}_{4}, 2.5 \mathrm{mM} \mathrm{NaCl}, 2 \mathrm{mM}$ $\mathrm{MgSO}_{4}, 0.45 \mathrm{mM} \mathrm{CaCl} 2,9 \mathrm{mM} \mathrm{FeSO}, 10 \mathrm{mM}$ glucose and $1 \mathrm{~L}$ water ( $\mathrm{pH} 6.9)$ ], complete medium (CM), or yeast extract peptone dextrose (YEPD) liquid medium $[1 \%(\mathrm{~g} / \mathrm{v})$ peptone, $0.3 \%(\mathrm{~g} / \mathrm{v})$ yeast extract, $2 \%(\mathrm{~g} / \mathrm{v})$ glucose and $1 \mathrm{~L}$ water ( $\mathrm{pH}$ 6.7)]; conidiation was assessed in carboxymethyl cellulose (CMC) liquid medium [0.1\% (g/v) car-

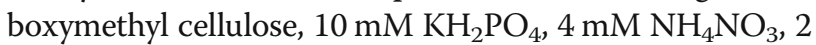
$\mathrm{mM} \mathrm{MgSO}_{4}$ and $1 \mathrm{~L}$ water] as described previously (Liu X et al. 2013). For the induction of DON biosynthesis, strains were grown in liquid trichothecene biosynthesis induction (TBI) medium (30 g sucrose, $1 \mathrm{~g}$ $\mathrm{KH}_{2} \mathrm{PO}_{4}, 0.5 \mathrm{~g} \mathrm{MgSO}_{4} \cdot 7 \mathrm{H}_{2} \mathrm{O}, 0.5 \mathrm{~g} \mathrm{KCl}, 0.01 \mathrm{~g} \mathrm{FeS}-$ $\mathrm{O}_{4} \cdot 7 \mathrm{H}_{2} \mathrm{O}, 1.47 \mathrm{~g}$ putrescine hydrochloride, trace elements and $1 \mathrm{~L}$ water $(\mathrm{pH} 4.5)]$ at $25^{\circ} \mathrm{C}$ in the dark (Gardiner et al. 2009).

\section{Generation of gene deletion mutants}

Gene deletion and complementation strains were generated using a protocol described previously ( $\mathrm{Yu}$ et al. 2014). The primers used to amplify the flanking sequences for each gene are listed in Additional file 1: Table S1. The resulting PCR products were transformed into protoplasts of the wild-type strain $\mathrm{PH}-1$, as described previously (Hou et al. 2002). Putative gene deletion transformants were selected on PDA medium supplemented with hygromycin $(100 \mu \mathrm{g} / \mathrm{mL})$, identified by PCR assays with the relevant primers (Additional file 1: Table S1) and further confirmed by Southern blot assays. To complement the $\Delta$ Fgpsd2 mutation, PCR products including the native promoter, open reading fragment of the gene and a geneticin resistance cassette were fused and transformed into the $\Delta F g p s d 2$ mutant. Transformants were selected by geneticin $(100 \mu \mathrm{g} / \mathrm{mL})$, identified by PCR and designated $\triangle F g p s d 2-C$. The complementation strains $\triangle F g$ cho2-C and $\triangle$ Fgopi3- $C$ were constructed via a similar approach. All mutants generated in this study were preserved in $15 \%$ glycerol at $-80{ }^{\circ} \mathrm{C}$.

\section{Fungicide sensitivity and stress response determination}

To assess fungal growth under environmental stress conditions, mycelial plugs (5 $\mathrm{mm}$ in diameter) taken from the periphery of a 3-day-old colony of each strain were inoculated on $\mathrm{CM}$ amended with the following compounds: carbendazim $(0.3 \mu \mathrm{g} / \mathrm{mL})$, phenamacril $(0.25 \mu \mathrm{g} / \mathrm{mL})$, tebuconazole $(0.25 \mu \mathrm{g} / \mathrm{mL})$, or rapamycin $(0.025 \mu \mathrm{g} / \mathrm{mL})$. To determine the sensitivities of these mutants to osmotic or cell wall stress agents, fresh mycelia were inoculated on CM amended with the following products: Paraquat $(100 \mu \mathrm{g} / \mathrm{mL})$ and $0.05 \% \mathrm{H}_{2} \mathrm{O}_{2}$, caffeine $(0.75 \mu \mathrm{g} / \mathrm{mL})$, SDS $(10 \mu \mathrm{g} / \mathrm{mL})$, Congo Red $(0.3 \mu \mathrm{g} / \mathrm{mL})$, or calcofluor white (CFW, $100 \mu \mathrm{g} / \mathrm{mL})$. The growth rescue experiment was conducted on MM amended with $0.04 \%(\mathrm{v} / \mathrm{v})$ ethanolamine (Etn), choline $(5 \mathrm{mM})$ and serine $(1 \mathrm{mM})$. After the plates were incubated at $25^{\circ} \mathrm{C}$ for 3 days, images were obtained, and the colony diameters on each plate were measured. Each experiment was repeated independently three times.

\section{Conidiation assay}

The conidiation of each strain was induced in CMC liquid medium, and conidia were collected for later research. Briefly, fresh mycelia of each strain $(50 \mathrm{mg})$ taken from the periphery of a 3-day-old colony were inoculated in a $250 \mathrm{~mL}$ flask containing $100 \mathrm{~mL}$ of $\mathrm{CMC}$ medium. The flasks were incubated at $25^{\circ} \mathrm{C}$ for 4 days in a shaker $(180 \mathrm{rpm})$. For each strain, the number of conidia in the broth was determined using a hemacytometer. In addition, the strains were incubated on CMC agar plates. The conidial morphology and lengths and the number of septa were observed with a confocal microscope after CFW staining.

\section{Virulence detection assay}

Conidia of each strain were resuspended in sterile water to a concentration of $1 \times 10^{5}$ conidia $/ \mathrm{mL}$. A $10 \mu \mathrm{L}$ aliquot of conidial suspension was injected into a floret in the central spikelet of a single flowering wheat head of the susceptible cultivar Jimai 22. There were ten replicates for each strain. After inoculation, the plants were maintained at $22 \pm 2{ }^{\circ} \mathrm{C}$ under $95 \%-100 \%$ humidity. Fifteen days after inoculation, the number of infected spikelets in each inoculated wheat head was recorded. The experiment was repeated three times.

\section{DON production assay}

Fresh mycelia of each strain were induced in TBI liquid medium at $28^{\circ} \mathrm{C}$ with agitation $(180 \mathrm{rpm})$ and were then filtered with gauze on the seventh day after incubation. The DON content in each sample was extracted and measured by a Waters 1525 HPLC system (Palo Alto,CA, USA) as described previously (Liu et al. 2013). The experiment was repeated independently three times.

\section{Confocal microscopy and image analysis}

A $7.5 \mathrm{mM}$ stock solution of FM4-64 (catalog no. T13320; $10 \times 100 \mu$ g; Invitrogen) was prepared by dissolving $100 \mu \mathrm{g}$ of FM4-64 in $108 \mu \mathrm{L}$ of DMSO. A working solution of $7.5 \mu \mathrm{M}$ was prepared by diluting $1 \mu \mathrm{L}$ of the stock solution in $1 \mathrm{~mL}$ of water. Confocal microscopy was performed with a Zeiss LSM 780 Meta laser 
scanning confocal microscope. FM4-64 was excited using a $515 \mathrm{~nm}$ laser and emission was observed at 640 $\mathrm{nm}$. CFW was excited using a $395 \mathrm{~nm}$ laser and emission was observed at $440 \mathrm{~nm}$. Images were processed using Zen software (Black edition, version 10.0, Zeiss).

\section{Additional files}

\section{Additional file 1: Table S1. A list of PCR primers used in this study. (DOCX $18 \mathrm{~kb}$ )}

Additional file 2: Figure S1. Schematic representation of gene disruption strategy and Southern blot analyses of the deletion mutants. a Schematic representation of the disruption strategy for Fgpsd2, Fgcho2, Fgopi3, Fgect1, Fgcpt1, Fgept1 and Fgcki1. Black arrows indicate hygromycin resistance gene $(H P H)$. b PCR assays for identification of the gene deletion mutants. c Southern blot analyses of the mutants. A fragment of $\mathrm{HPH}$ gene was used as the probe in the Southern blot assays. The restriction enzyme used for digestion of genomic DNA preparation was indicated in schematic representation of the disruption strategy for each strain. The size of the resulting hybridization band was indicated for each strain at the bottom of Southern blot images. (TIF $8725 \mathrm{~kb}$ )

Additional file 3: Figure S2. Relative mycelial growth rate of mutants on completed medium to that of the wild-type $\mathrm{PH}-1$. Strains were grown on $\mathrm{CM}$ at $25^{\circ} \mathrm{C}$ for 3 days. Line bars in each column denote standard deviations of three repeated experiments. Bars followed by the same letter are not significantly different according to LSD test at $P=0.01$. (TIF $2364 \mathrm{~kb}$ )

Additional file 4: Figure S3. Phenotypes of complementation mutants in the de novo pathway. a Conidial morphology of the wild-type and complementation mutants. The septa were stained with calcofluor white and observed by fluorescent microscope. Bar $=25 \mu \mathrm{m}$. $\mathbf{b}$ The number of conidia of each strain after 4 days of incubation in $100 \mathrm{~mL}$ CMC medium. c Conidial length of the wild-type and complementation mutants. d Mycelial growth inhibition of the wild-type and mutants towards each antifungal agent. Strains were grown on the CM amended without or with tebuconazole, carbendazim, phenamacril or rapamycin at the concentration indicated in the material and methods. e Mycelial growth inhibition rate of mutants towards antifungal agents. Line bars in each column denote standard deviations of three repeated experiments. Bars followed by the same letter are not significantly different according to LSD test at $P=0.01$. (TIF $10888 \mathrm{~kb}$ )

\section{Abbreviations}

CDP-Cho: Cytidyldiphosphatecholine; CDP-DAG: Cytidyldiphosphate diacylglycerol; CDP-Etn: Cytidyldiphosphate-ethanolamine; Cho: Choline; ChoP: Choline phosphate; Etn: Ethanolamine; EtnP: Ethanolamine phosphate; OMM: Outer mitochondrial membrane; PC: Phosphatidylcholine; PE: Phosphatidylethanolamine; PS: Phosphatidylserine

\section{Acknowledgements}

We would like to acknowledge the anonymous reviewers for their work on this paper.

\section{Funding}

This research was supported by the International Science \& Technology Cooperation Program of China (2016YFE0112900), the National Key R \& D Plan (2017YFC1600904), the National Natural Science Fund for Distinguished Young Scholar (31525020) and the Young Elite Scientist Sponsorship Program (2017QNRC001).

\section{Availability of data and materials}

The datasets used and analyzed during the current study are available from the corresponding author on reasonable request.

\section{Authors' contributions}

YC and ZM conceived and designed the experiment. JW, HX, CZ and TW performed the experiment and analyzed the data. JW, CY and ZM checked all the data. JW, CY and ZM wrote the manuscript. All authors read and approved the final manuscript.

\section{Ethics approval and consent to participate}

Not applicable.

\section{Consent for publication}

Not applicable.

\section{Competing interests}

The authors declare that they have no competing interests.

\section{Author details}

${ }^{1}$ Institute of Biotechnology, Zhejiang University, 866 Yuhangtang Road, Hangzhou 310058, China. ${ }^{2}$ Key Laboratory of Molecular Biology of Crop Pathogens and Insects, Zhejiang University, 866 Yuhangtang Road, Hangzhou 310058, China. 'S tate Key Laboratory of Rice Biology, Zhejiang University, 866 Yuhangtang Road, Hangzhou 310058, China.

Received: 2 January 2019 Accepted: 12 April 2019

Published online: 29 April 2019

\section{References}

Akhberdi O, Zhang Q, Wang HC, Li YY, Chen LF, Wang D, et al. Roles of phospholipid methyltransferases in pycnidia development, stress tolerance and secondary metabolism in the taxol-producing fungus Pestalotiopsis microspore. Microbiol Res. 2018;210:33-42.

Audenaert K, Callewaert E, Hofte M, De Saeger S, Haesaert G. Hydrogen peroxide induced by the fungicide prothioconazole triggers deoxynivalenol (DON) production by Fusarium graminearum. BMC Microbiol. 2010;10:112.

Bergo MO, Gavino BJ, Steenbergen R, Sturbois B, Parlow AF, Sanan DA, et al. Defining the importance of phosphatidylserine synthase 2 in mice. J Biol Chem. 2002;277:47701-8.

Birner R, Burgermeister M, Schneiter R, Daum G. Roles of phosphatidylethanolamine and of its several biosynthetic pathways in Saccharomyces cerevisiae. Mol Biol Cell. 2001;12:997-1007.

Blandino M, Haidukowski M, Pascale M, Plizzari L, Scudellari D, Reyneri A. Integrated strategies for the control of Fusarium head blight and deoxynivalenol contamination in winter wheat. Field Crop Res. 2012;133:139-49.

Braun BR, Hoog MV, d'Enfert C, Martchenko M, Dungan J, Kuo A, et al. A humancurated annotation of the Candida albicans genome. PLoS Genet. 2005;1:36-57.

Bukata L, Altabe S, de Mendoza D, Ugalde RA, Comerci DJ. Phosphatidylethanolamine synthesis is required for optimal virulence of Brucella abortus. J Bacteriol. 2008;190:8197-203.

Burgermeister M, Birner-Grunberger R, Nebauer R, Daum G. Contribution of different pathways to the supply of phosphatidylethanolamine and phosphatidylcholine to mitochondrial membranes of the yeast Saccharomyces cerevisiae. BBA-Mol Cell Biol Lipids. 2004;1686:161-8.

Cassilly CD, Reynolds TB. PS, it's complicated: the roles of phosphatidylserine and phosphatidylethanolamine in the pathogenesis of Candida albicans and other microbial pathogens. J Fungi. 2018;1:28.

Chen Y, Wang J, Yang R, Ma Z. Current situation and management strategiesof Fusarium head blight in China. Plant Protection. 2017;5:11-7 (in Chinese).

Chen Y, Zheng SY, Ju ZZ, Zhang CQ, Tang GF, Wang J, et al. Contribution of peroxisomal docking machinery to mycotoxin biosynthesis, pathogenicity and pexophagy in the plant pathogenic fungus Fusarium graminearum. Environ Microbiol. 2018a;20:3224-45.

Chen YL, Montedonico AE, Kauffman S, Dunlap JR, Menn FM, Reynolds TB. Phosphatidylserine synthase and phosphatidylserine decarboxylase are essential for cell wall integrity and virulence in Candida albicans. Mol Microbiol. 2010;75:1112-32.

Chen YX, Li B, Cen K, Lu YZ, Zhang SW, Wang CS. Diverse effect of phosphatidylcholine biosynthetic genes on phospholipid homeostasis, cell autophagy and fungal developments in Metarhizium robertsii. Environ Microbiol. 2018b;20:293-304.

Daum G, Lees ND, Bard M, Dickson R. Biochemistry, cell biology and molecular biology of lipids of Saccharomyces cerevisiae. Yeast. 1998;14:1471-510.

Desjardins AE, Proctor RH, Bai GH, McCormick SP, Shaner G, Buechley G, et al. Reduced virulence of trichothecene-nonproducing mutants of Gibberella zeae in wheat field tests. Mol Plant-Microbe Interact. 1996;9:775-81. 
Gao Q, Lu YZ, Yao HY, Xu YJ, Huang W, Wang CS. Phospholipid homeostasis maintains cell polarity, development and virulence in Metarhizium robertsii. Environ Microbiol. 2016;18:3976-90.

Gardiner DM, Kazan K, Manners JM. Nutrient profiling reveals potent inducers of trichothecene biosynthesis in Fusarium graminearum. Fungal Genet Biol. 2009:46:604-13.

Ghannoum MA, Rice LB. Antifungal agents: mode of action, mechanisms of resistance, and correlation of these mechanisms with bacterial resistance. Clin Microbiol Rev. 1999;12:501-17

Gibellini F, Smith TK. The Kennedy pathway--De novo synthesis of phosphatidylethanolamine and phosphatidylcholine. IUBMB Life. 2010;62:414-28.

Gulshan K, Schmidt JA, Shahi P, Moye-Rowley WS. Evidence for the bifunctional nature of mitochondrial phosphatidylserine decarboxylase: role in Pdr3-dependent retrograde regulation of PDR5 expression. Mol Cell Biol. 2008;28:5851-64.

Gulshan K, Shahi P, Moye-Rowley WS. Compartment-specific synthesis of phosphatidylethanolamine is required for normal heavy metal resistance. Mol Biol Cell. 2010;21:443-55.

Guo Y, Au WC, Shakoury-Elizeh M, Protchenko O, Basrai M, Prinz WA, et al. Phosphatidylserine is involved in the ferrichrome-induced plasma membrane trafficking of Arn1 in Saccharomyces cerevisiae. J Biol Chem. 2010;285:39564-73.

Hasim S, Vaughn EN, Donohoe D, Gordon DM, Pfiffner S, Reynolds TB. Influence of phosphatidylserine and phosphatidylethanolamine on farnesol tolerance in Candida albicans. Yeast. 2018;35:343-51.

Hou Z, Xue C, Peng Y, Katan T, Kistler HC, Xu JR. A mitogen-activated protein kinase gene (MGV1) in Fusarium graminearum is required for female fertility, heterokaryon formation, and plant infection. Mol Plant-Microbe Interact. 2002;15:1119-27.

Kent C. Eukaryotic phospholipid biosynthesis. Annu Rev Biochem. 1995;64:315-43.

Khandelwal NK, Sarkar P, Gaur NA, Chattopadhyay A, Prasad R. Phosphatidylserine decarboxylase governs plasma membrane fluidity and impacts drug susceptibilities of Candida albicans cells. BBA-Biomembranes. 2018;1860:2308-19.

Lee S, Uchida Y, Emoto K, Umeda M, Kuge O, Taguchi T, et al. Impaired retrograde membrane traffic through endosomes in a mutant $\mathrm{CHO}$ cel defective in phosphatidylserine synthesis. Genes Cells. 2012;17:728-36.

Lehmann S, Serrano M, L'Haridon F, Tjamos SE, Metraux JP. Reactive oxygen species and plant resistance to fungal pathogens. Phytochemistry. 2015;112:54-62.

Letts VA, Klig LS, Bae-Lee M, Carman GM, Henry SA. Isolation of the yeast structural gene for the membrane-associated enzyme phosphatidylserine synthase. Proc Natl Acad Sci U S A. 1983;80:7279-83.

Liu X, Jiang J, Yin YN, Ma ZH. Involvement of FgERG4 in ergosterol biosynthesis, vegetative differentiation and virulence in Fusarium graminearum. Mol Plant Pathol. 2013;14:71-83.

Magan N, Hope R, Colleate A, Baxter ES. Relationship between growth and mycotoxin production by Fusarium species, biocides and environment. Eur J Plant Pathol. 2002;108:685-90.

Matsuo Y, Fisher E, Patton-Vogt J, Marcus S. Functional characterization of the fission yeast phosphatidylserine synthase gene, pps1, reveals novel cellular functions for phosphatidylserine. Eukaryot Cell. 2007;6:2092-101.

Milus EA, Parsons CE. Evaluation of foliar fungicides for controlling Fusarium head blight of wheat. Plant Dis. 1994;78:697-9.

Minder AC, de Rudder KEE, Narberhaus F, Fischer HM, Hennecke H, Geiger O. Phosphatidylcholine levels in Bradyrhizobium japonicum membranes are critical for an efficient symbiosis with the soybean host plant. Mol Microbiol. 2001;39:1186-98

Nagle JF, Tristram-Nagle S. Structure of lipid bilayers. Biochim Biophys Acta. 2000;1469:36.

Pan J, Hu CT, Yu JH. Lipid biosynthesis as an antifungal target. J Fungi. 2018;4(2):50.

Proctor RH, Hohn TM, McCormick SP. Reduced virulence of Gibberella zeae caused by disruption of a trichothecene toxin biosynthetis gene. Mol PlantMicrobe Interact. 1995;8:593-601.

Seong KY, Pasquali M, Zhou XY, Song J, Hilburn K, McCormick S, et al. Global gene regulation by Fusarium transcription factors Tri6 and Tri10 reveals adaptations for toxin biosynthesis. Mol Microbiol. 2009;72:354-67.

Simpson DR, Weston GE, Turner JA, Jennings P, Nicholson P. Differential control of head blight pathogens of wheat by fungicides and consequences for mycotoxin contamination of grain. Eur J Plant Pathol. 2001;107:421-31.

Sturbois-Balcerzak B, Stone SJ, Sreenivas A, Vance JE. Structure and expression of the murine phosphatidylserine synthase-1 gene. J Biol Chem. 2001;276:8205-12

Subramaniam S, Fahy E, Gupta S, Sud M, Byrnes RW, Cotter D, et al. Bioinformatics and systems biology of the lipidome. Chem Rev. 2011; 111:6452-90.
Sud M, Fahy E, Cotter D, Brown A, Dennis EA, Glass CK, et al. LMSD: Lipid maps structure database. Nucleic Acids Res. 2007;35:D527-D32.

Tang GF, Chen Y, Xu JR, Kistler HC, Ma ZH. The fungal myosin I is essential for Fusarium toxisome formation. PLoS Pathog. 2018b;14(1):e1006827.

Tang GF, Zhang CQ, Ju ZZ, Zheng SY, Wen ZY, Xu SD, et al. The mitochondrial membrane protein FgLetm1 regulates mitochondrial integrity, production of endogenous reactive oxygen species and mycotoxin biosynthesis in Fusarium graminearum. Mol Plant Pathol. 2018a;19:1595-611.

Tao L, Gao N, Chen SF, Yu JH. The choC gene encoding a putative phospholipid methyltransferase is essential for growth and development in Aspergillus nidulans. Curr Genet. 2010;56:283-96.

Trotter PJ, Voelker DR. Identification of a nonmitochondrial phosphatidylserine decarboxylase activity (Psd2) in the yeast Saccharomyces cerevisiae. J Biol Chem. 1995;270:6062-70.

Vallejo MC, Nakayasu ES, Longo LVG, Ganiko L, Lopes FG, Matsuo AL, et al. Lipidomic analysis ofextracellular vesicles from the pathogenic phase of Paracoccidioides brasiliensis. PLoS One. 2012;7(6):e39463.

Van Meer G, Voelker DR, Feigenson GW. Membrane lipids: where they are and how they behave. Nat Rev Mol Cell Bio. 2008;9:112-24.

Vance JE, Steenbergen R. Metabolism and functions of phosphatidylserine. Prog Lipid Res. 2005:44:207-34.

Welti R, Li WQ, Li MY, Sang YM, Biesiada H, Zhou HE, et al. Profiling membrane lipids in plant stress responses - role of phospholipase D alpha in freezinginduced lipid changes in Arabidopsis. J Biol Chem. 2002;277:31994-2002.

Wessel M, Klusener S, Godeke J, Fritz C, Hacker S, Narberhaus F. Virulence of Agrobacterium tumefaciens requires phosphatidylcholine in the bacterial membrane. Mol Microbiol. 2006;62:906-15.

Wolf JM, Espadas J, Luque-Garcia J, Reynolds T, Casadevall A. Lipid biosynthetic genes affect Candida albicans extracellular vesicle morphology, cargo, and immunostimulatory properties. Eukaryot Cell. 2015;14:745-54.

Xu XM, Nicholson P. Community ecology of fungal pathogens causing wheat head blight. Annu Rev Phytopathol. 2009;47:83-103.

Yu F, Gu Q, Yun Y, Yin Y, Xu JR, Shim WB, Ma Z. The TOR signaling pathway regulates vegetative development and virulence in Fusarium graminearum. New Phytol. 2014;203:219-32.

Yun Y, Liu Z, Yin Y, Jiang J, Chen Y, Xu JR, et al. Functional analysis of the Fusarium graminearum phosphatome. New Phytol. 2015;207:119-34.

Zhang LG, Jia XJ, Chen CJ, Zhou MG. Characterization of carbendazim sensitivity and trichothecene chemotypes of Fusarium graminearum in Jiangsu Province of China. Physiol Mol Plant Pathol. 2013;84:53-60.

Zhang YJ, Yu JJ, Zhang YN, Zhang X, Cheng CJ, Wang JX, et al. Effect of Carbendazim resistance on trichothecene production and aggressiveness of Fusarium graminearum. Mol Plant-Microbe Interact. 2009;22:1143-50.

Zinser E, Sperka-Gottlieb CD, Fasch EV, Kohlwein SD, Paltauf F, Daum G. Phospholipid synthesis and lipid composition of subcellular membranes in the unicellular eukaryote Saccharomyces cerevisiae. J Bacteriol. 1991;173:2026-34.

\section{Ready to submit your research? Choose BMC and benefit from:}

- fast, convenient online submission

- thorough peer review by experienced researchers in your field

- rapid publication on acceptance

- support for research data, including large and complex data types

- gold Open Access which fosters wider collaboration and increased citations

- maximum visibility for your research: over $100 \mathrm{M}$ website views per year

At $\mathrm{BMC}$, research is always in progress.

Learn more biomedcentral.com/submissions 